\title{
Adaptive Predefined Performance Neural Control for Robotic Manipulators with Unknown Dead Zone
}

\author{
Shifen Shao, ${ }^{1}$ Kaisheng Zhang $\mathbb{D}^{1},{ }^{1} \mathrm{Jun} \mathrm{Li}^{2}$ and Jirong Wang ${ }^{3}$ \\ ${ }^{1}$ College of Electrical and Control Engineering, Shaanxi University of Science and Technology, Xi'an 710021, China \\ ${ }^{2}$ College of Computer Science and Technology, Qingdao University, Qingdao 266071, China \\ ${ }^{3}$ College of Mechanical and Electronic Engineering, Qingdao University, Qingdao 266071, China
}

Correspondence should be addressed to Kaisheng Zhang; qdhgysfwz@163.com

Received 6 January 2020; Accepted 3 February 2020; Published 12 May 2020

Guest Editor: Jing $\mathrm{Na}$

Copyright (c) 2020 Shifen Shao et al. This is an open access article distributed under the Creative Commons Attribution License, which permits unrestricted use, distribution, and reproduction in any medium, provided the original work is properly cited.

This paper proposes an adaptive predefined performance neural control scheme for robotic manipulators in the presence of nonlinear dead zone. A neural network $(\mathrm{NN})$ is utilized to estimate the model uncertainties and unknown dynamics. An improved funnel function is designed to guarantee the transient behavior of the tracking error. The proposed funnel function can release the assumption on the conventional funnel control. Then, an adaptive predefined performance neural controller is proposed for robotic manipulators, while the tracking errors fall within a prescribed funnel boundary. The closed-loop system stability is proved via Lyapunov function. Finally, the numerical simulation results based on a 2-DOF robotic manipulator illustrate the control effect of the presented approach.

\section{Introduction}

Robotic manipulators have been widely utilized in industrial applications such as manufacturing industry, aerospace, and military equipment [1-9]. Nevertheless, the nonlinear terms include the nonlinear friction, model uncertainties, and dead zone that can reduce the control accuracy. To address this problem, the conventional PID controller was designed for robotic manipulators, but PID cannot achieve the satisfactory control performance [9]. To improve the tracking performance of robotic manipulators, a variety of control strategies were proposed for robotic systems such as adaptive control [10,11], nonlinear control [12], and backstepping control [13-15].

In fact, the difficulties in the control design for robotic systems mainly stem from nonlinear terms. To tackle these nonlinear terms, disturbance observer techniques were proposed to reject the unknown disturbance $[1,16,17]$. In [1], a new unknown dynamics estimator- (UDE-) based firstorder filter is proposed for robotic manipulators, the UDE was incorporated into control design that can effectively reject the unknown dynamics. An unknown input observer
(UIO) was developed by introducing the first-order filter to estimate the unknown dynamics of servomechanisms, where the UIO had only one tuned parameter [18]. A novel nonlinear disturbance observer (NDO) was proposed for robotic manipulators in [17]. A disturbance observer (DOB) was devised for robot manipulators, where the external disturbance can be rejected by using the DOB [19]. Although the aforementioned disturbance observer-based control strategies can improve the performance of the robotic manipulator, the transient behavior is not considered in control design.

On the other hand, as neural networks (NNs) [1, 20-25] or fuzzy logic systems (FLS) [26-28] have been used to approximate the system uncertainties due to their approximation ability. In [25], an adaptive neural network control was proposed for robot manipulators, where the $\mathrm{NN}$ was utilized to approximate the unknown dead zone and system uncertainties. In [29], a contouring control method was proposed for robot manipulators and the $\mathrm{NN}$ was used to estimate the unknown dynamics. In [30], a NN-based terminal sliding mode control (TSMC) was designed for robot manipulators with actuator dynamics, where the $\mathrm{NN}$ was 
used to estimate the unknown actuator dynamics. A neuralfuzzy control was used to estimate the inverse dynamics; then, the approximation was incorporated into an adaptive neural-fuzzy controller to compensate the unknown dynamics of robot systems [31]. Although the aforementioned approaches can improve the control performance, the transient behavior and steady-state performance are not considered in controller design.

Recently, it is well known that the prescribed performance control (PPC) method can be used to quantitatively analyse the transient behavior [32]. The main feature is that a prescribed function with maximum overshoot and convergence rate is used to transform the original tracking error into a new error. Then, the new error is used to design a controller in which the tracking error can be remained within a predefined boundary. This control method has been used to control some systems [33-38]. In [39], an adaptive prescribed performance control was proposed for servomechanisms to improve the control performance. In [40], a modified prescribed performance function was proposed and incorporated into control design to control piezo-actuated positioning systems. A PPC was developed to control a variable stiffness actuated robot in [34]. Moreover, a funnel control (FC) as a constraint control was also proposed to guarantee the transient response [41]. The concept of FC is to construct an adjustable proportional gain $\tau$ to control the dynamics systems. The funnel control has been used to control some practice systems such as twomass systems [42], air-breathing hypersonic vehicles [43], and nonlinear dynamics systems [44]. In [45], a funnel control based on the adaptive fuzzy control was proposed to control stochastic nonlinear systems, where the fuzzy logic is utilized to approximate the unknown nonlinear dynamics. A neural network based on the adaptive control was developed for two-mass systems with backlash, where the neural network was employed to estimate the unknown backlash [46]. If a control system is with high relative degree $(r \geq 3)$, the funnel control may not suit this kind of systems. Thus, the application of funnel control is limited. Moreover, the application of funnel control in robotic manipulators cannot be found.

This paper will propose a novel adaptive neural prescribed performance control method for robotic manipulators with unknown dead zone. A novel funnel variable is defined based on the tracking error. The modified funnel variable can release the assumption on the original funnel control. An echo state neural network (ESN) is adopted to estimate the unknown dynamics of robotic manipulators, and the approximation is used in control design to compensate the nonlinear dead zone. Then, an adaptive control scheme for a robotic manipulator is proposed to improve the control performance. Numerical simulation demonstrates the effectiveness of the proposed control approach.

The special contributions of this paper are as follows:

(i) A novel funnel function is proposed based on the tracking error, and it can release the limitation on the original funnel function and is used in control design to improve the control performance (ii) A neural network is utilized to estimate the nonlinear dead zone, and the approximation is to design a controller, where the dead zone is compensated

(iii) The effectiveness of the proposed control method is evaluated based on a robotic manipulator by using numerical simulations

The remainder of this paper is organized as follows. Section 2 presents system description, funnel control design, and echo state neural network structure. An adaptive neural funnel controller is shown in Section 3. Numerical simulation results are given in Section 4. Finally, the paper is concluded in Section 5.

\section{Problem Formulation}

2.1. System Description. This paper considers a $n$-degree-offreedom (DOF) robotic manipulator, which can be modeled as

$$
M(q) \ddot{q}+C(q, \dot{q}) \dot{q}+G(q)+d_{l}=d(\tau),
$$

where $q, \dot{q}$, and $\ddot{q}$ are the robot joint position, velocity, and acceleration, respectively; $M(q)$ denotes the inertia matrix, $C(q, \dot{q})$ represents the Coriolis/centripetal torque, including the viscous friction and nonlinear damping, $G(q)$ is the gravity torque, $\tau$ is the control input, and $d_{l}$ is the unknown disturbance.

For the matrices $M(q)$ and $C(q, \dot{q})$, the following properties hold.

Property 1. The matrix $\dot{M}(q)-2 C(q, \dot{q})$ is a skew-symmetric matrix.

Property 2. The matrix $M(q)$ is bounded such that $0<M_{a} \leq\|M(q)\| \leq M_{b}$, where $M_{a}$ and $M_{b}$ are positive constants.

Assumption 1. The dead-zone nonlinearity (see Figure 1) can be written as

$$
d(\tau)= \begin{cases}d_{r}(\tau), & \text { if } \tau(t) \geq b_{r}, \\ 0, & \text { if } b_{l}<v(t)<b_{r}, \\ d_{l}(\tau), & \text { if } \tau(t) \leq b_{l},\end{cases}
$$

where $\tau(t)$ is the control torque, $d_{l}(v)$ and $d_{r}(v)$ denote unknown smooth functions, and $b_{l}<0$ and $b_{r}>0$ denote constants.

$$
\begin{aligned}
& d_{r}(\tau(t)) \text { and } d_{l}(\tau(t)) \text { can be written as } \\
& d_{l}(v)=d_{l}(v)-d_{l}\left(b_{l}\right) d_{l}^{\prime}\left(\xi_{l}\right)\left(v-b_{l}\right), \\
& \forall v \in\left(-\infty, b_{l}\right] \text { with } \xi_{l} \in\left(-\infty, b_{l}\right), \\
& d_{r}(v)=d_{r}(v)-d_{r}\left(b_{r}\right)=d_{r}^{\prime}\left(\xi_{r}\right)\left(v-b_{r}\right), \\
& \forall v \in\left[b_{r}+\infty\right) \text { with } \xi_{l} \in\left(b_{r},+\infty\right),
\end{aligned}
$$

where $d_{i}^{\prime}=\mathrm{d}\left(D_{i} \xi\right) /\left.\mathrm{d} \xi\right|_{\xi=\xi_{i}}, i=l, r$, denotes the derivative of $d_{i}(\xi), i=l, r$.

Using (3) and (8), the dead zone is given as

$$
d(\tau(t))=\left(\chi_{l}(t)+\chi_{r}(t)\right) \tau(t)+\rho(t)=d(t) \tau(t)+\rho(t),
$$




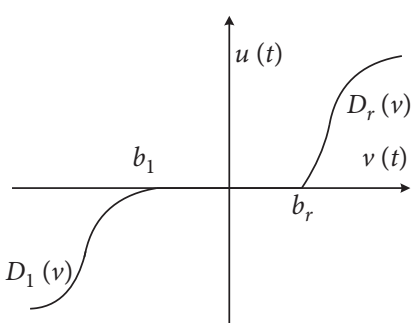

Figure 1: Nonlinear dead zone.

where $d(t)=\chi_{l}(t)+\chi_{r}(t)$ with

$$
\begin{aligned}
& \chi_{l}(t)= \begin{cases}d_{l}^{\prime}\left(\xi_{l}^{\prime}\right), & \text { if } \tau(t) \leq b_{r}, \\
0, & \text { if } \tau(t)>b_{r},\end{cases} \\
& \chi_{r}(t)= \begin{cases}d_{r}^{\prime}\left(\xi_{r}^{\prime}\right), & \text { if } \tau(t) \leq b_{l}, \\
0, & \text { if } \tau(t)>b_{l} .\end{cases}
\end{aligned}
$$

2.2. Echo State Neural Network Approximation. The echo state neural network is a novel NN with superior capability to approximate the unknown dynamics. The basic architecture of the ESN is shown in Figure 2. The ESN is composed of three parts: (1) $K$ input neurons, $N$ reservoir neurons, and $L$ output layer. The ESN model can written as

$$
\begin{aligned}
\dot{X} & =C\left(-a X+f\left(W^{\text {in } u}+W X+W^{\text {back }} y\right)\right), \\
y & =G\left(W_{0}^{T} X\right),
\end{aligned}
$$

where $X$ denotes the reservoir neuron state, $C>0$ is a time constant, and a represents the leaking decay rate. $W^{\text {in }} \in R^{N \times K}, W \in R^{N \times N}$, and $W^{\text {out }} \in R^{N \times L}$ denote the input weight matrix, the reservoir weight matrix, and the feedback weight matrix, respectively. The ESN can be used to approximate any continuous function $f(x)$ over a compact domain $\Omega \in R^{m}$.

The function $f(x)$ can be expressed as

$$
f(x)=W^{T} \Phi(x)+\varepsilon, \quad \forall x \in \Omega \subset R^{m},
$$

where $\varepsilon$ is the estimation error of the $\mathrm{ESN},|\varepsilon| \leq \varepsilon_{m}$, and $W$ denotes the weight.

Therefore,

$$
W^{*}=\arg \min _{W \in R^{L}}\left\{\sup _{x \in \Omega}\left|f(x)-W^{* T} X(x)\right|\right\} .
$$

2.3. Funnel Control. Funnel control [41] is a novel control strategy. By the error transformation, the original tracking error can be transformed into a new error dynamics. Then, the new errors are used to design a control that can guarantee the control error remaining within a predefined boundary.

The system $S$ has the following properties:

(i) Relative degree $r=1$ or 2

(ii) Minimum phase

(iii) Known high frequency gain

The controller is given as

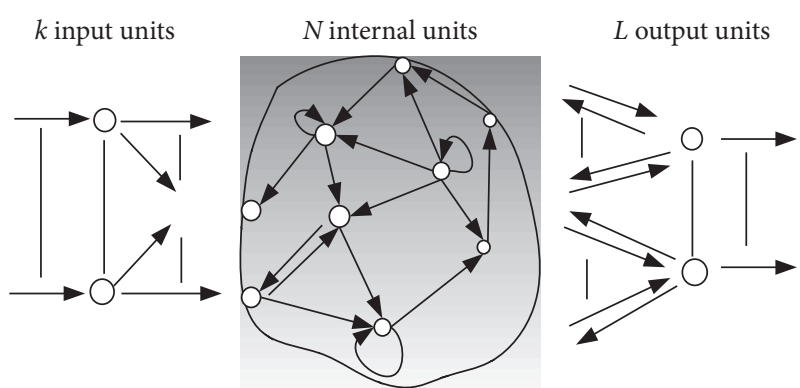

FIgURE 2: Basic architecture of the ESN.

$$
u(t)=\tau\left(F_{\varphi}(t), \psi(t),\|e(t)\|\right) \cdot e(t),
$$

where $F_{\varphi}(t)$ is the funnel function and $\psi(t)$ denotes the scaling factor. The distance $d_{v}(t)$ is defined as

$$
d_{v}(t)=F_{\varphi}(t)-\|e(t)\|,
$$

where $e(t)$ is the tracking error, which is defined as

$$
e(t)=x_{d}-x(t) \text {. }
$$

Thus, the funnel itself is defined as the set

$$
F_{\varphi}^{\prime}:=\left\{(t, e) \in R \times R^{n} \mid \varphi(t) \cdot\|e(t)\|<1\right\} .
$$

The gain $\tau(\cdot)$ is

$$
\tau\left(F_{\varphi}(t), \psi(t),\|e(t)\|\right)=\frac{\psi(t)}{F_{\varphi}(t)-\|e(t)\|} .
$$

According to [41], the boundary (see Figure 3 ) is

$$
F_{\varphi}(t)=\varphi_{0} \cdot \exp (-a t)+\varphi_{\infty},
$$

where $\varphi_{0}, \varphi_{\infty}$, and $a$ are design parameters and satisfy $\varphi_{0} \geq \varphi_{\infty}>0$ and $|e(0)|<F_{\varphi}(0)=\varphi_{0}+\varphi_{\infty}$.

A novel funnel variable can be given as

$$
z(t)=\frac{e(t)}{F_{\varphi}(t)-\|e(t)\|} .
$$

\section{Adaptive Control Design}

3.1. Controller Design. In this section, we consider the full state information, $x_{1}$ and $x_{2}$, is measured, where $x_{1}=\left[q_{1}, q_{2}, \ldots, q_{n}\right]^{T}$ and $x_{2}=\left[\dot{q}_{1}, \dot{q}_{2}, \ldots, \dot{q}_{n}\right]^{T}$. (Figure 4) Then, the system model can be expressed as

$$
\begin{aligned}
& \dot{x}_{1}=x_{2}, \\
& \dot{x}_{2}=M^{-1}\left[D(\tau)-C\left(x_{1}, x_{2}\right) x_{2}-G(x-1)-d_{l}\right] .
\end{aligned}
$$

Step 1. The tracking error $e_{1}$ is defined as

$$
e_{1}=x_{1}-x_{d}
$$

where $x_{d}$ is the desired trajectory. According to (15), the funnel error can be defined as

$$
z_{1}=\frac{e_{1}}{F_{\varphi 1}-\left|e_{1}\right|} .
$$




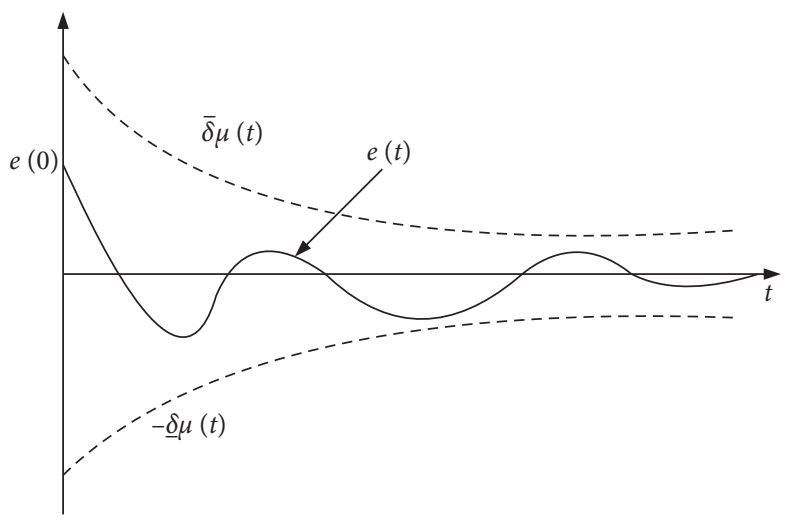

Figure 3: Funnel control.

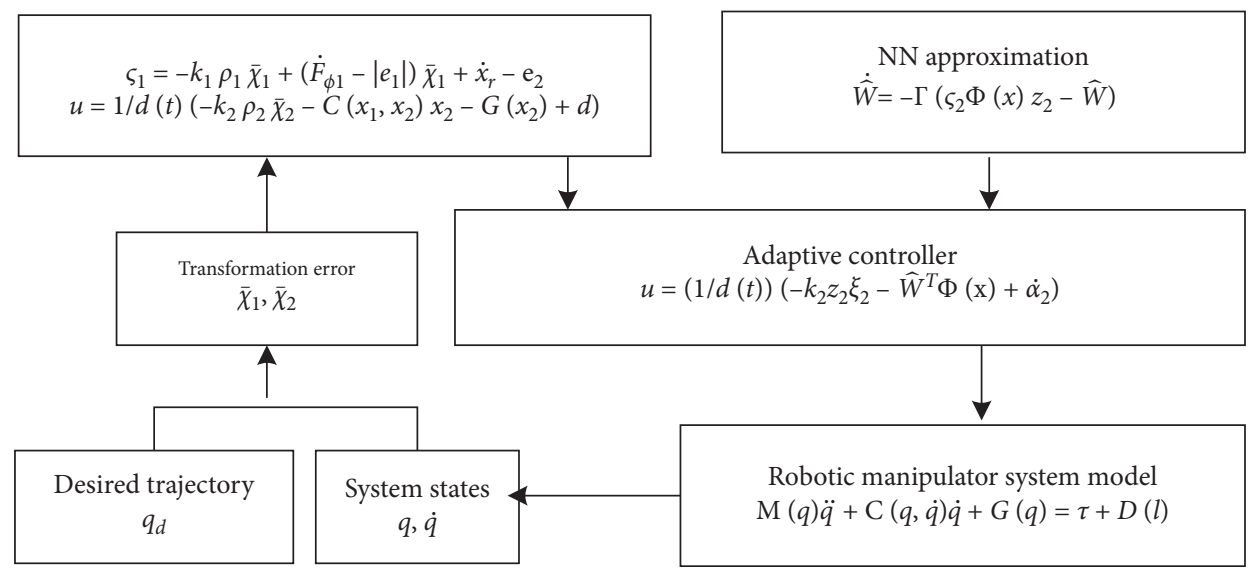

FIgURE 4: Controller architecture.

The time derivative of (20) is

$$
\begin{aligned}
\dot{z}_{1} & =\frac{1}{\left(F_{\varphi 1}-\left|e_{1}\right|\right)^{2}}\left[\dot{e}_{1}\left(F_{\varphi 1}-\left|e_{1}\right|\right)-e_{1}\left(\dot{F}_{\varphi 1}-\left|\dot{e}_{1}\right|\right)\right] \\
& =\frac{1}{\left(F_{\varphi 1}-\left|e_{1}\right|\right)}\left[x_{2}-\left(\dot{F}_{\varphi 1}-\left|\dot{e}_{1}\right|\right) z_{1}-\dot{x}_{d}\right] .
\end{aligned}
$$

The Lyapunov function is defined as

$$
V_{1}=\frac{1}{2} z_{1}^{2}
$$

Its time derivative is

$\dot{V}_{1}=z_{1} \dot{z}_{1}=\frac{z_{1}}{\left(F_{\varphi 1}-\left|e_{1}\right|\right)}\left[x_{2}-\left(\dot{F}_{\varphi 1}-\left|\dot{e}_{1}\right|\right) z_{1}-\dot{x}_{d}\right]$.

The second error variable is defined as

$$
e_{2}=x_{2}-\alpha_{2} \text {. }
$$

Substituting (24) into (23), one has

$$
\begin{aligned}
\dot{V}_{1} & =z_{1} \dot{z}_{1}=\frac{z_{1}}{\left(F_{\varphi 1}-\left|e_{1}\right|\right)}\left[\alpha_{2}+e_{2}-\left(\dot{F}_{\varphi 1}-\left|\dot{e}_{1}\right|\right) z_{1}-\dot{x}_{d}\right] \\
& =z_{1} \zeta_{1}\left[\alpha_{2}+e_{2}-\left(\dot{F}_{\varphi 1}-\left|\dot{e}_{1}\right|\right) z_{1}-\dot{x}_{d}\right],
\end{aligned}
$$

where $\zeta_{1}=1 /\left(F_{\varphi 1}-\left|e_{1}\right|\right)$.
An intermediate control signal is chosen as

$$
\alpha_{2}=-k_{1} z_{1} \zeta_{1}+\left(\dot{F}_{\varphi 1}-\left|\dot{e}_{1}\right|\right) z_{1}+\dot{x}_{d}-e_{2},
$$

where $k_{1}$ is a design parameter.

Step 2. The time derivative of $e_{2}$ is

$$
\dot{e}_{2}=\dot{x}_{2}-\dot{\alpha}_{2} \text {. }
$$

According to (17), the second funnel error variable can be defined as

$$
z_{2}=\frac{e_{2}}{F_{\varphi 2}-\left|e_{2}\right|} .
$$

The time derivative of $z_{2}$ is

$$
\begin{aligned}
\dot{z}_{2}= & \frac{1}{\left(F_{\varphi 1}-\left|e_{2}\right|\right)^{2}}\left[\dot{e}_{2}\left(F_{\varphi 2}-\left|e_{2}\right|\right)-e_{2}\left(\dot{F}_{\varphi 2}-\left|\dot{e}_{2}\right|\right)\right] \\
= & \frac{1}{\left(F_{\varphi 2}-\left|e_{2}\right|\right)}\left[\dot{x}_{2}-\left(\dot{F}_{\varphi 2}-\left|\dot{e}_{2}\right|\right) z_{2}-\dot{\alpha}_{2}\right] \\
= & \zeta_{2} z_{2}\left[M ^ { - 1 } \left(D(\tau)-C\left(x_{1}, x_{2}\right) x_{2}-G\left(x_{1}\right)\right.\right. \\
& \left.\left.-d_{l}\right)-\left(\dot{F}_{\varphi 2}-\left|\dot{e}_{2}\right|\right) z_{2}-\dot{\alpha}_{2}\right]
\end{aligned}
$$

where $\zeta_{2}=1 /\left(F_{\varphi 2}-\left|e_{2}\right|\right)$. 
The Lyapunov function is defined as

$$
V_{2}=\frac{1}{2} z_{2}^{T} M\left(x_{1}\right) z_{2} \text {. }
$$

The derivative of (30) is

$$
\begin{aligned}
\dot{V}_{2}= & z_{2}^{T} M\left(x_{1}\right) \dot{z}_{2}+z_{2}^{T} \frac{1}{2} \dot{M}\left(x_{1}\right) z_{2} \\
= & \zeta_{2} z_{2}^{T}\left[\left(d(t) v(t)+\rho(t)-C\left(x_{1}, x_{2}\right) x_{2}-G\left(x_{1}\right)-d_{l}\right)\right. \\
& \left.-\left(\dot{F}_{\varphi 2}-\left|\dot{e}_{2}\right|\right) z_{2}-\dot{\alpha}_{2}+\frac{1}{2} \dot{M}\left(x_{1}\right) z_{2}\right] \\
= & \zeta_{2} z_{2}^{T}\left[\left(d(t) v(t)+F(x)-\dot{\alpha}_{2}\right] .\right.
\end{aligned}
$$

where $F(x)=\rho(t)-C\left(x_{1}, x_{2}\right) x_{2}-G\left(x_{1}\right)-d_{l^{-}}\left(\dot{F}_{\varphi 2}-\left|\dot{e}_{2}\right|\right)+$ $z_{2}(1 / 2) \dot{M}\left(x_{1}\right) z_{2}$ denotes the unknown term, which can be approximated by using the $\mathrm{NN}$.

The actual controller can be designed as

$$
\tau(t)=\frac{1}{d(t)}\left(-k_{2} z_{2} \zeta_{2}+\widehat{W} \Phi(X)+\dot{\alpha}_{2}\right)
$$

where $\widehat{W}$ denotes the estimation of $W$, which is defined as

$$
\dot{\widehat{W}}=-\Gamma\left(\zeta_{2} \Phi(X) z_{2}-\sigma \widehat{W}\right)
$$

where $\Gamma$ and $\sigma$ are design parameters.

3.2. Stability Analysis. In this section, we will employ the Lyapunov function to analyse the convergence of the closedloop system.

Theorem 1. Consider the robotic manipulators (1) with the proposed controller (32), intermediate controller (26), and adaptive law (33), then all the signals of the closed-loop system are bounded, and the tracking error can converge to the prescribed zone.

Proof. A Lyapunov function is chosen as

$$
V=V_{1}+V_{2} \text {. }
$$

The time derivative of (34) is

$$
\dot{V}=\dot{V}_{1}+\dot{V}_{2} \text {. }
$$

Substituting (23) and (31) into (35), one has

$$
\begin{aligned}
\dot{V}= & z_{1} \zeta_{1}\left[\alpha_{2}+e_{2}-\left(\dot{F}_{\varphi 1}-\left|\dot{e}_{1}\right|\right) z_{1}-\dot{x}_{d}\right] \\
& +\zeta_{2} z_{2}\left[M^{-1}\left(D(\tau)-C\left(x_{1}, x_{2}\right) x_{2}-G\left(x_{1}\right)-d_{l}\right)\right. \\
& \left.-\left(\dot{F}_{\varphi 2}-\left|\dot{e}_{2}\right|\right) z_{2}-\dot{\alpha}_{2}\right] .
\end{aligned}
$$

Based on (27), (33), and adaptive law (34), one has

$$
\dot{V}=-k_{1} \zeta_{1} z_{1}-k_{2} \zeta_{2} z_{2}+\sigma \widetilde{W} \widehat{W} .
$$

Using Young's inequality, one has

$$
\sigma \widetilde{W}^{T} \widehat{W} \leq-\frac{\sigma}{2} \widetilde{W}^{T} \tilde{W}+\frac{\sigma}{2} W^{2}
$$

Substituting (38) into (37), we have

$$
\begin{aligned}
\dot{V} & \leq-k_{1} \zeta_{1} z_{1}-k_{2} \zeta_{2} z_{2}-\frac{\sigma}{2} \widetilde{W}^{T} \widetilde{W}+\frac{\sigma}{2} W^{2} \\
& \leq-\rho V+\delta
\end{aligned}
$$

where $\rho=\min \left\{2 k_{1} \zeta_{1}, 2 k_{2} \zeta_{2}, \sigma\right\}$ and $\delta=(\sigma / 2) W^{2}$ are positive constants.

From (39), we know that $V$ is bounded by $\delta / \rho$. Therefore, all the signals of the closed-loop system are semiglobally uniformly and ultimately bounded.

The parameter tuning guidelines are given as follows:

(1) Select the funnel variables $\varphi_{0}, \varphi_{\infty}$, and $a$, and they should satisfy the initial conditions $\varphi_{0}(0)>\varphi_{\infty}(0)>0$.

(2) Choose the control gains $k_{1}$ and $k_{2}$, and the adaptive law parameters are $\Gamma$ and $\sigma$. In general, they can be set large for the ease of fast convergence. However, practical control systems do not allow using large gains because they may produce oscillations. Hence, they can be chosen based on a trial-and-error method.

\section{Numerical Simulation}

In this section, we will employ an example to illustrate the control performance of the developed control method. A diagram of the robotic manipulator system with 2-DOF is shown in Figure 5. The robotic manipulator parameters are listed in Table 1.

The system matrices $M(q), C(q, \dot{q})$, and $G(q)$ are defined as

$$
\begin{aligned}
& M(q)=\left[\begin{array}{cc}
\alpha l_{1}^{2}+\beta l_{2}^{2}+2 \gamma l_{1} l_{2} c_{2} & \beta l_{2}^{2}+\gamma l_{1} l_{2} c_{2} \\
\beta l_{2}^{2}+\gamma l_{1} l_{2} c_{2} & \beta l_{2}^{2}
\end{array}\right], \\
& G(q)=0, \\
& C(q)=\left[\begin{array}{cc}
-2 \gamma l_{1} l_{2} \dot{q}_{2} s_{2} & -\gamma l_{1} l_{2} \dot{q}_{2} s_{2} \\
\gamma l_{1} l_{2} \dot{q}_{2} s_{2} & 0
\end{array}\right],
\end{aligned}
$$

where $\alpha=1 / 4 m_{1}+m_{2}+m_{3}+m_{4}, \quad \beta=1 / 4 m_{3}+m_{4}$, and $\gamma=1 / 2 m_{3}+m_{4}$, respectively.

The controller parameters are given as $k_{1}=5$ and $k_{2}=10$. The adaptive parameters are $\Gamma=10 I$ and $\sigma=0.02$. The initial weight $\widehat{W}=0$. The initial position of robotic manipulator is chosen as $q_{0}=[0,0]$ and $\dot{q}_{0}=[0,0]$. The friction term is $F(q)=\left[15 \dot{q}_{1}+6 \operatorname{sign}\left(\dot{q}_{1}\right) ; 15 \dot{q}_{2}+\right.$ $\left.6 \operatorname{sign}\left(\dot{q}_{2}\right)\right]$. The funnel function is $F_{\varphi i}(t)=\varphi_{0 i} \cdot \exp \left(-a_{i} t\right)+$ $\varphi_{\infty i}, i=1,2$, with $\varphi_{01}=\varphi_{02}=0.18, \varphi_{\infty 1}=\varphi_{\infty 22}=0.01$, and $a_{1}=a_{2}=2$. The reference signals are given as $q_{1 d}=0.3 \sin t$ and $q_{2 d}=0.3 \sin t$.

Figures 6-8 depict the simulation results, where the output tracking performance, control actions, and ESN estimation are given. From Figure 6, we can see that the developed control approach can achieve the satisfactory control performance. In addition, we can see that the 


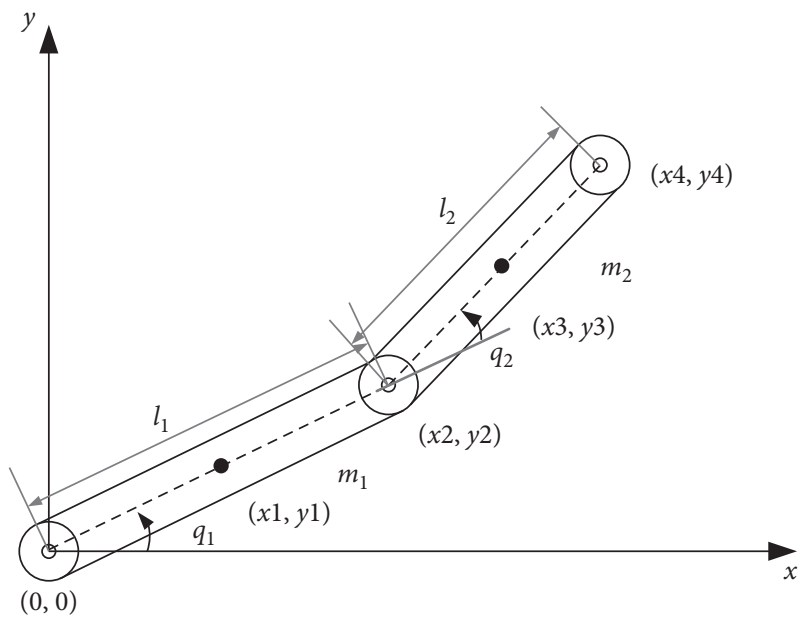

FIgURE 5: Diagram of the robotic manipulator.

TABle 1: Parameters for the robotic manipulator.

\begin{tabular}{lccc}
\hline Parameters & Description & Value & Unit \\
\hline$l_{1}$ & Length of link 1 & 1 & $\mathrm{~m}$ \\
$l_{2}$ & Length of link 2 & 0.8 & $\mathrm{~m}$ \\
$m_{1}$ & Mass of link 1 & 1 & $\mathrm{~kg}$ \\
$m_{2}$ & Mass of joint 2 & 1.5 & $\mathrm{~kg}$ \\
$m_{3}$ & Mass of link 2 & 2 & $\mathrm{~kg}$ \\
$m_{3}$ & Mass of actuator & 1.5 & $\mathrm{~kg}$ \\
\hline
\end{tabular}
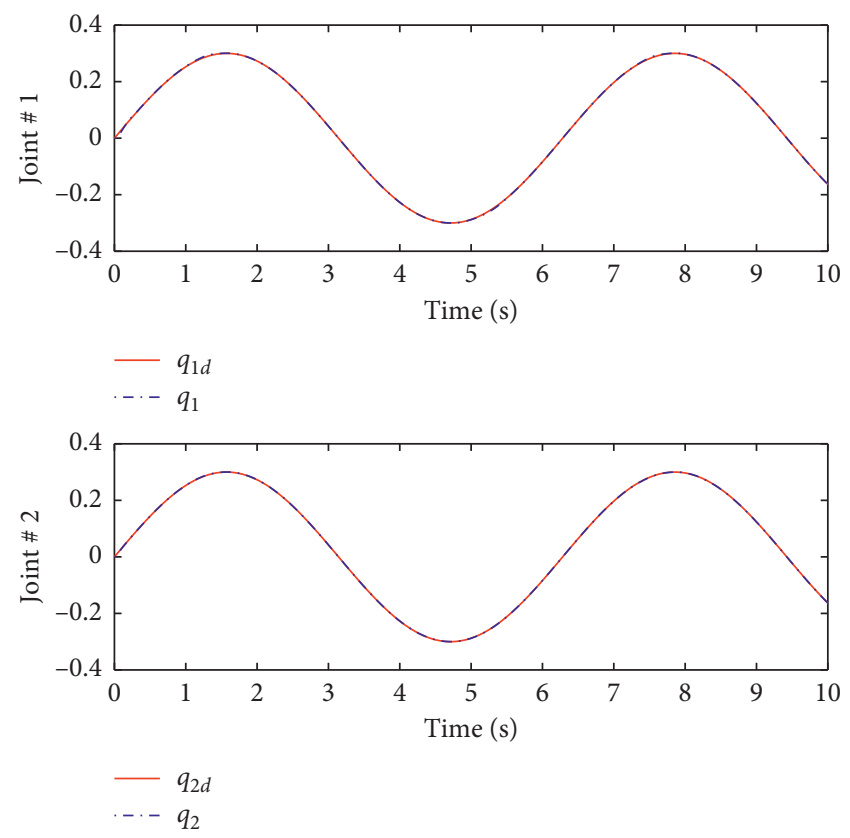

FIgURE 6: Output tracking.

nonlinear friction can be estimated by using the echo state neural network. From these results, we find that the proposed control method improves the tracking performance of the robotic manipulator.
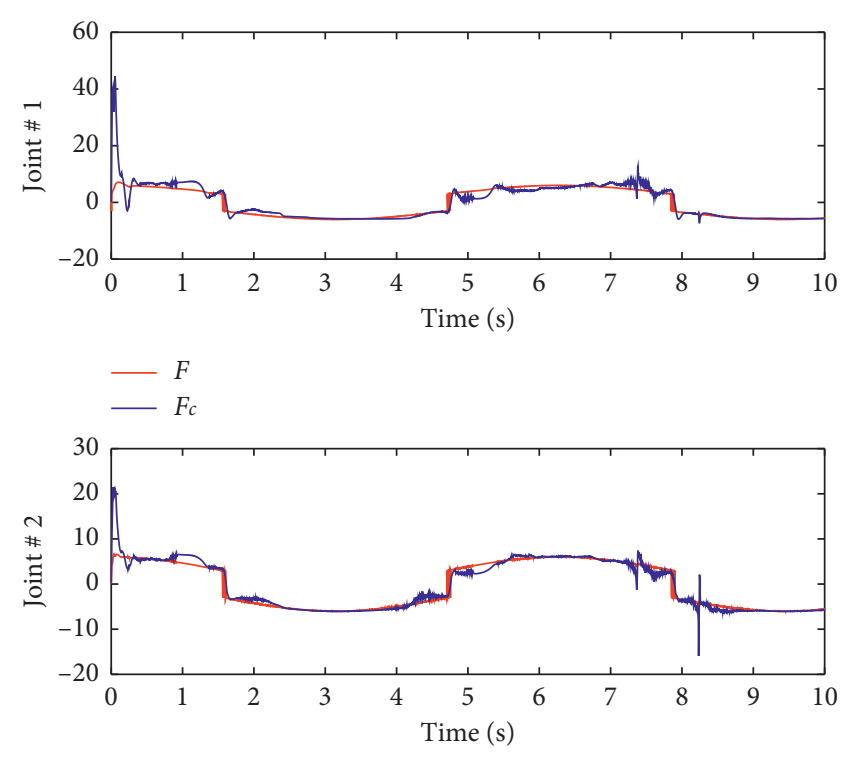

$-F$

$-F_{c}$

Figure 7: Friction compensation.
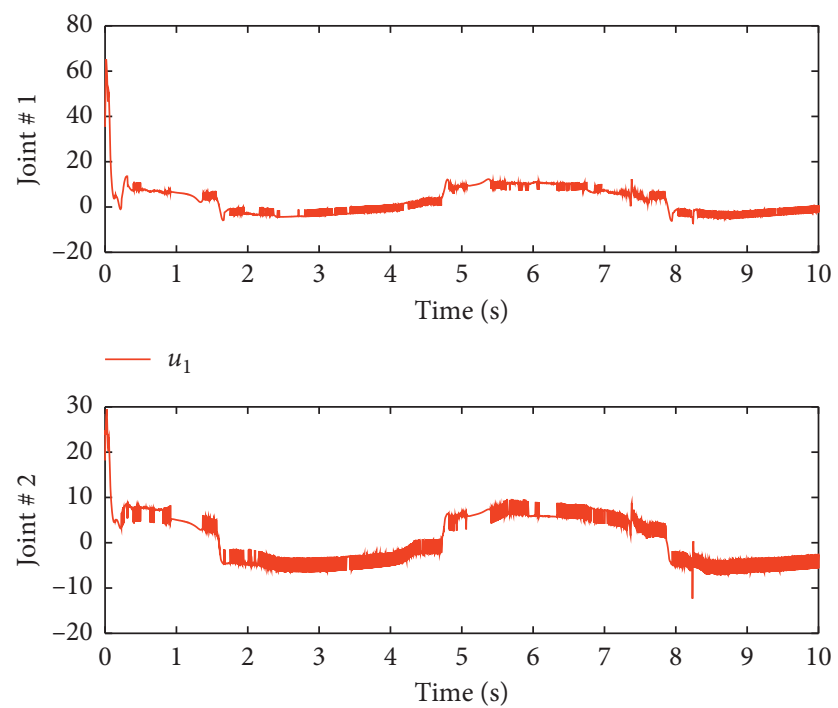

$-u_{2}$

Figure 8: Control signals.

\section{Conclusion}

In this paper, an adaptive predefined performance control for robotic manipulators in the presence of nonlinear dead zone was proposed. A novel funnel variable was designed based on the tracking error. The new error variable was utilized to design a controller that can guarantee the transient response. A neural network was adopted to estimate the unknown dynamics (parameter uncertainties and nonlinear dead zone), and the approximation was utilized in controller design to compensate the unknown dynamics. An adaptive controller based on funnel control was designed for the robotic manipulator. Both the transient response and 
steady-state performance of the tracking error are guaranteed.

\section{Data Availability}

The data used to support the findings of this study are available from the corresponding author upon request.

\section{Conflicts of Interest}

The authors declare that they have no conflicts of interest.

\section{Acknowledgments}

This work was supported by the Project of Science and Technology Department of Shanxi Province (16JK1100).

\section{References}

[1] J. Na, J. Yang, S. Wang, G. Gao, and C. Yang, "Unknown dynamics estimator-based output-feedback control for nonlinear pure-feedback systems," IEEE Transactions on Systems, Man, and Cybernetics: Systems, pp. 1-12, 2019.

[2] S. Wang, X. Ren, J. Na, and T. Zeng, "Extended-state-observer-based funnel control for nonlinear servomechanisms with prescribed tracking performance," IEEE Transactions on Automation Science and Engineering, vol. 14, no. 1, pp. 98108, 2017.

[3] T. Li, S. Duan, J. Liu, L. Wang, and T. Huang, "A spintronic memristor-based neural network with radial basis function for robotic manipulator control implementation," IEEE Transactions on Systems, Man, and Cybernetics: Systems, vol. 46, no. 4, pp. 582-588, 2016.

[4] W. He, Y. Ouyang, and J. Hong, "Vibration control of a flexible robotic manipulator in the presence of input deadzone," IEEE Transactions on Industrial Informatics, vol. 13, no. 1, pp. 48-59, 2017.

[5] H. Gao, W. He, C. Zhou, and C. Sun, "Neural network control of a two-link flexible robotic manipulator using assumed mode method," IEEE Transactions on Industrial Informatics, vol. 15, no. 2, pp. 755-765, 2019.

[6] M. Jafarinasab, S. Sirouspour, and E. Dyer, "Model-based motion control of a robotic manipulator with a flying multirotor base," IEEE/ASME Transactions on Mechatronics, vol. 24, no. 5, pp. 2328-2340, 2019.

[7] S. Wang, J. Na, and Y. Xing, "Adaptive optimal parameter estimation and control of servo mechanisms: theory and experiments," IEEE Transactions on Industrial Electronics, p. 1, 2020.

[8] Q. Chen, S. Xie, M. Sun, and X. He, "Adaptive nonsingular fixed-time attitude stabilization of uncertain spacecraft," IEEE Transactions on Aerospace and Electronic Systems, vol. 54, no. 6, pp. 2937-2950, 2018.

[9] Y. Jia, "Robust control with decoupling performance for steering and traction of $4 \mathrm{WS}$ vehicles under velocity-varying motion," IEEE Transactions on Control Systems Technology, vol. 8, no. 3, pp. 554-569, 2000.

[10] C. Yang, Y. Jiang, W. He, J. Na, Z. Li, and B. Xu, "Adaptive parameter estimation and control design for robot manipulators with finite-time convergence," IEEE Transactions on Industrial Electronics, vol. 65, no. 10, pp. 8112-8123, 2018.

[11] M. Tao, Q. Chen, X. He, and M. Sun, "Adaptive fixed-time fault-tolerant control for rigid spacecraft using a double power reaching law," International Journal of Robust and Nonlinear Control, vol. 29, no. 12, pp. 4022-4040, 2019.

[12] R. M. Robinson, C. S. Kothera, R. M. Sanner, and N. M. Wereley, "Nonlinear control of robotic manipulators driven by pneumatic artificial muscles," IEEE/ASME Transactions on Mechatronics, vol. 21, no. 1, pp. 55-68, 2016.

[13] N. Nikdel, M. Badamchizadeh, V. Azimirad, and M. A. Nazari, "Fractional-order adaptive backstepping control of robotic manipulators in the presence of model uncertainties and external disturbances," IEEE Transactions on Industrial Electronics, vol. 63, no. 10, pp. 6249-6256, 2016.

[14] S. Wang, H. Yu, and J. Yu, "Robust adaptive tracking control for servo mechanisms with continuous friction compensation," Control Engineering Practice, vol. 87, pp. 76-82, 2019.

[15] S. Wang, J. Na, H. Yu, and Q. Chen, "Finite time parameter estimation-based adaptive predefined performance control for servo mechanisms," ISA Transactions, vol. 87, pp. 174-186, 2019.

[16] J. N. Yun, J. Su, Y. I. Kim, and Y. C. Kim, "Robust disturbance observer for two-inertia system," IEEE Transactions on Industrial Electronics, vol. 60, no. 7, pp. 2700-2710, 2013.

[17] W.-H. Chen, D. J. Ballance, P. J. Gawthrop, and J. O’Reilly, “A nonlinear disturbance observer for robotic manipulators," IEEE Transactions on Industrial Electronics, vol. 47, no. 4, pp. 932-938, 2000.

[18] S. Wang, J. Na, X. Ren, H. Yu, and J. Yu, "Unknown input observer-based robust adaptive funnel motion control for nonlinear servomechanisms," International Journal of Robust and Nonlinear Control, vol. 28, no. 18, pp. 6163-6179, 2018.

[19] S. P. Chan, "A disturbance observer for robot manipulators with application to electronic components assembly," IEEE Transactions on Industrial Electronics, vol. 42, no. 5, pp. 487-493, 1995.

[20] S. Wang, J. Na, and X. Ren, "Rise-based asymptotic prescribed performance tracking control of nonlinear servo mechanisms," IEEE Transactions on Systems, Man, and Cybernetics: Systems, vol. 48, no. 12, pp. 2359-2370, 2018.

[21] S. Wang, H. Yu, J. Yu, J. Na, and X. Ren, "Neural-networkbased adaptive funnel control for servo mechanisms with unknown dead-zone," IEEE Transactions on Cybernetics, vol. 50, no. 4, pp. 1383-1394, 2020.

[22] J. Na, S. Wang, Y.-J. Liu, Y. Huang, and X. Ren, "Finite-time convergence adaptive neural network control for nonlinear servo systems," IEEE Transactions on Cybernetics, pp. 1-12, 2019.

[23] Q. Chen, H. Shi, and M. Sun, "Echo state network-based backstepping adaptive iterative learning control for strictfeedback systems: an error-tracking approach," IEEE Transactions on Cybernetics, pp. 1-14, 2019.

[24] S. Wang, Q. Chen, X. Ren, and H. Yu, "Neural network-based adaptive funnel sliding mode control for servo mechanisms with friction compensation," Neurocomputing, vol. 377, pp. 16-26, 2020.

[25] W. He, A. O. David, Z. Yin, and C. Sun, "Neural network control of a robotic manipulator with input deadzone and output constraint," IEEE Transactions on Systems, Man, and Cybernetics: Systems, vol. 46, no. 6, pp. 759-770, 2016.

[26] L. Cao, H. Li, N. Wang, and Q. Zhou, "Observer-based eventtriggered adaptive decentralized fuzzy control for nonlinear large-scale systems," IEEE Transactions on Fuzzy Systems, vol. 27, no. 6, pp. 1201-1214, 2019.

[27] T. Wang, S. Tong, J. Yi, and H. Li, "Adaptive inverse control of cable-driven parallel system based on type-2 fuzzy logic 
systems," IEEE Transactions on Fuzzy Systems, vol. 23, no. 5, pp. 1803-1816, 2015.

[28] Y. Li and S. Tong, "Adaptive fuzzy control with prescribed performance for block-triangular-structured nonlinear systems," IEEE Transactions on Fuzzy Systems, vol. 26, no. 3, pp. 1153-1163, 2018.

[29] L. Wang, T. Chai, and C. Yang, "Neural-network-based contouring control for robotic manipulators in operational space," IEEE Transactions on Control Systems Technology, vol. 20, no. 4, pp. 1073-1080, 2012.

[30] L. Wang, T. Chai, and L. Zhai, "Neural-network-based terminal sliding-mode control of robotic manipulators including actuator dynamics," IEEE Transactions on Industrial Electronics, vol. 56, no. 9, pp. 3296-3304, 2009.

[31] F. Sun, L. Li, H.-X. Li, and H. Liu, "Neuro-fuzzy dynamicinversion-based adaptive control for robotic manipulators-discrete time case," IEEE Transactions on Industrial Electronics, vol. 54, no. 3, pp. 1342-1351, 2007.

[32] C. P. Bechlioulis and G. A. Rovithakis, "Prescribed performance adaptive control for multi-input multi-output affine in the control nonlinear systems," IEEE Transactions on Automatic Control, vol. 55, no. 5, pp. 1220-1226, 2010.

[33] Q. Hu, X. Shao, and L. Guo, "Adaptive fault-tolerant attitude tracking control of spacecraft with prescribed performance," IEEE/ASME Transactions on Mechatronics, vol. 23, no. 1, pp. 331-341, 2018.

[34] E. Psomopoulou, A. Theodorakopoulos, Z. Doulgeri, and G. A. Rovithakis, "Prescribed performance tracking of a variable stiffness actuated robot," IEEE Transactions on Control Systems Technology, vol. 23, no. 5, pp. 1914-1926, 2015.

[35] Y. Zhu, J. Qiao, and L. Guo, "Adaptive sliding mode disturbance observer-based composite control with prescribed performance of space manipulators for target capturing," IEEE Transactions on Industrial Electronics, vol. 66, no. 3, pp. 1973-1983, 2019.

[36] C. Hua, G. Liu, L. Li, and X. Guan, “Adaptive fuzzy prescribed performance control for nonlinear switched time-delay systems with unmodeled dynamics," IEEE Transactions on Fuzzy Systems, vol. 26, no. 4, pp. 1934-1945, 2018.

[37] J. Na, Y. Huang, Q. Pei, X. Wu, G. Gao, and G. Li, “Active suspension control of full-car systems without function approximation," IEEE/ASME Transactions on Mechatronics, vol. 25, no. 2, pp. 779-791, 2020.

[38] Y. Huang, J. Na, X. Wu, and G. Gao, “Approximation-free control for vehicle active suspensions with hydraulic actuator," IEEE Transactions on Industrial Electronics, vol. 65, no. 9, pp. 7258-7267, 2018.

[39] J. Na, Q. Chen, X. Ren, and Y. Guo, “Adaptive prescribed performance motion control of servo mechanisms with friction compensation," IEEE Transactions on Industrial Electronics, vol. 61, no. 1, pp. 486-494, 2014.

[40] M. L. Nguyen, X. Chen, and F. Yang, "Discrete-time quasisliding-mode control with prescribed performance function and its application to piezo-actuated positioning systems," IEEE Transactions on Industrial Electronics, vol. 65, no. 1, pp. 942-950, 2018.

[41] A. Ilchmann, E. P. Ryan, and C. J. Sangwin, "Tracking with prescribed transient behaviour," ESAIM: Control, Optimisation and Calculus of Variations, vol. 7, Article ID 471493, 2002.

[42] A. Ilchmann and H. Schuster, "Pi-funnel control for two mass systems," IEEE Transactions on Automatic Control, vol. 54, no. 4, pp. 918-923, 2009.
[43] X. Bu, "Air-breathing hypersonic vehicles funnel control using neural approximation of non-affine dynamics," IEEE/ ASME Transactions on Mechatronics, vol. 23, no. 5, pp. 2099-2108, 2018.

[44] S. I. Han and J. M. Lee, "Fuzzy echo state neural networks and funnel dynamic surface control for prescribed performance of a nonlinear dynamic system," IEEE Transactions on Industrial Electronics, vol. 61, no. 2, pp. 1099-1112, 2014.

[45] C. Hua, K. Li, and X. Guan, "Event-based dynamic output feedback adaptive fuzzy control for stochastic nonlinear systems," IEEE Transactions on Fuzzy Systems, vol. 26, no. 5, pp. 3004-3015, 2018.

[46] S. Wang, H. Yu, J. Yu, and X. Gao, "Adaptive neural funnel control for nonlinear two-inertia servo mechanisms with backlash," IEEE Access, vol. 7, pp. 33 338-33 345, 2019. 